\title{
Germanica
}

GERMANICA $38 \mid 2006$

Voix étrangères en langue allemande

\section{Heimatlose Weltliteratur? Zum Werk von Aglaja Veteranyi}

Littérature apatride et mondiale? À propos de l'ouvre littéraire d'Aglaja

Veteranyi

Laura Gieser

\section{(2) OpenEdition}

Journals

Édition électronique

URL : http://journals.openedition.org/germanica/409

DOI : 10.4000/germanica.409

ISSN : 2107-0784

Éditeur

Université de Lille

Édition imprimée

Date de publication : 1 juin 2006

Pagination : 63-85

ISBN : 2-913857-17-5

ISSN : 0984-2632

Référence électronique

Laura Gieser, « Heimatlose Weltliteratur? Zum Werk von Aglaja Veteranyi », Germanica [Online], 38 |

2006, Online erschienen am: 19 Februar 2010, abgerufen am 06 Oktober 2020. URL : http://

journals.openedition.org/germanica/409; DOI : https://doi.org/10.4000/germanica.409

Ce document a été généré automatiquement le 6 octobre 2020.

(c) Tous droits réservés 


\title{
Heimatlose Weltliteratur? Zum Werk von Aglaja Veteranyi
}

\author{
Littérature apatride et mondiale? À propos de l'œuvre littéraire d'Aglaja \\ Veteranyi
}

Laura Gieser

\section{Rumänien heißt nicht mehr Paris}

Mir wachsen fremde Sprachen an, zuerst die Fluchwörter, dann die Liebeserklärungen.

Gefällt es dir hier?

In allen Ländern wollen alle dasselbe wissen.

Nur in Rumänien fragt niemand danach, sagt die Tante. [...]

In Helvetia waren wir anerkannte Flüchtlinge.

Gefällt es Dir hier?

Auf diese Frage mussten wir dort immer mit JA antworten.

Ja, in Rumänien war es sehr schlimm.

Ja, hier ist es sehr schön. [...]

Auf unserem Ausländerausweis stand eine MITTEILUNG, die uns im Flüchtlingsbüro erklärt wurde.

Die Flüchtlingseigenschaft und die - wenn auch bloß vorübergehende - freiwillige Rückkehr in den Fluchtsstaat stehen in einem absoluten, nicht lösbaren inneren Widerspruch zueinander. Das eine muss das andere ausschließen.

Wenn der Tante oder der Mutter das Herz aus dem Mund wuchs, mussten sie es ausspucken.

Sonst stand uns nichts mehr im Weg für ein Leben wie die Menschen.

Mir wuchs das Herz nicht aus dem Mund.

Mir kam höchstens mein Cousin Schurubu in den Sinn. Schurubu hieß Schraube. In Rumänien war er mit mir unter den Tisch gekrochen. Später schnitt er sich einen Finger $a b^{1}$. 
1 Aglaja Veteranyis (1962-2002) Debütroman Warum das Kind in der Polenta kocht stellte die literarische Überraschung des Jahres 1999 dar. Der beeindruckende Roman erhielt die Ehrengabe des Kantons Zürich, den Kunstpreis Berlin sowie den Adelbert-von-Chamisso Förderpreis des Jahres $2000^{2}$. Es wurde eine aus Osteuropa stammende Schriftstellerin gewürdigt, die sich von Anfang an für die deutsche Sprache als poetisches Ausdrucksmittel entschieden hatte. Dem Roman waren bezeichnenderweise ein Lyrikband namens Geschenke. Ein Totentan $z^{3}$ sowie zahlreiche kleinere Veröffentlichungen in regionalen und überregionalen Literaturzeitschriften vorausgegangen. Ein weiterer Roman, Das Regal der letzten Atemzüge, erschien posthum im Jahre 2001 ebenso wie die 2005 veröffentlichte Kurzgeschichtensammlung Vom geräumten Meer, den gemieteten Socken und Frau Butter ${ }^{4}$.

2 Die Prosawerke Aglaja Veteranyis zeichnen sich formal durch eine lyrisch anmutende minimalistische Kürze und Verdichtung aus; inhaltlich sind die Erzählungen häufig autobiographisch bestimmt. Insbesondere die beiden Romane lassen sich als semifiktionale Mikro-Biographien lesen, wobei die Ich-Erzählersituation eine solche Annäherung zusätzlich suggeriert: «Alle unsere Familienfotos sahen wie Zirkusnummern aus, auch die Beerdigungen $»^{5}$. Beide Romane skizzieren tragischkomische Szenen aus dem Leben einer rumänischen Zirkusfamilie nach deren Flucht in den Westen und beide sind aus der Sicht einer namenlos bleibenden «Tochter» erzählt ${ }^{6}$.

In unseren Geschäftspausen bereitete sich ihr Mann im Schlaf auf seine Chefstelle vor, meine Mutter arbeitete als Serviertochter, und ich putzte Büros oder bewachte die Umkleidekabine in einem Warenhaus.

Ich wollte etwas anderes werden? ${ }^{7}$.

3 In beiden Fällen handelt es sich nicht nur um erinnernde Lebensbeschreibungen: Es wird darüber hinaus die doppelte Flucht eines Migrantenkindes inszeniert. Damit ist zum einen die Flucht des Kindes zusammen mit der Familie aus einem nationalen Gefängnis ins Ausland, zum anderen der persönliche Ausbruch des Kindes aus dem Ghetto der Familie (bzw. dem Wohnwagen als Heimat) in ein selbstbestimmtes Leben gemeint. Trotz aller destabilisierenden Effekte auf die Psyche der Betroffenen, wird die Grenzüberschreitung als Loslösung von festgefahrenen Strukturen und somit als wesentliche Voraussetzung dafür gesehen, dass ein Individuationsprozess beginnen kann, der, poetologisch betrachtet, gleichzeitig ein künstlerischer Entwicklungsprozess ist.

4 Wie bei vielen Autoren mit einem Migrationshintergrund, wird auch bei Aglaja Veteranyi die eigene Biographie im literarischen Text kreativ verarbeitet. Veteranyi flieht als fünfjähriges Kind 1967 zusammen mit einem Teil ihrer Familie aus der sozialistischen Diktatur Rumäniens in die Schweiz. Ihre nächsten Familienangehörigen und sie selbst sind Zirkusartisten: Für sie alle setzt nach der Flucht zunächst eine Phase der Wanderschaft durch die westliche Welt ein. Mehrsprachig aufgewachsen und weitgereist, gleichzeitig aber Analphabetin und ohne Schulbildung, kehrt die 17jährige Veteranyi nach dem Zusammenbruch des familiären Zusammenhalts - «Meine Familie ist im Ausland wie Glas zerbrochen $»^{8}-$ in die Schweiz zurück. Aglaja Veteranyi lässt sich in Zürich nieder. Dort lernt sie zum ersten Mal Lesen und Schreiben, wobei Deutsch ihre erste Schriftsprache wird.

5 Die Selbstkonstituierung als Autorin deutscher Sprache lässt Aglaja Veteranyi den bedrückenden und durchaus vulgären biographischen Hintergrund, die in der Kindheit und frühen Jugend erfahrene Vernachlässigung und Gewalt, in ihren Werken 
keineswegs beschönigen oder verschweigen'. Vielmehr sind es gerade diese Erfahrungen, die, gepaart mit einer zunächst desillusionierenden Begegnung mit dem Westen - «Das Glück hatte ich mir anders vorgestellt ${ }^{10}$ » -, den individuellen Überlebens- und Ausdruckswillen der Autorin gefördert zu haben scheinen. Die Autorin zitiert im Rahmen ihrer häufig grotesk zugespitzten Familienerzählung die in Bezug auf den unterentwickelten Staat des vormaligen Ostblocks dominierenden Bilder von politischer Unterdrückung und von Korruption, von Armut, Kindermissbrauch und von Menschenhandel. Selbst auf die Gefahr hin, damit westliche Klischees von Rumänien zu bedienen, scheut sie sich nicht, der Demoralisierung nachzugehen, die das Leben in einem totalitären Staat bestimmt und deren Folgen im zwischenmenschlichen Umgang - insbesondere als allgegenwärtiges, isolierendes Misstrauen - noch in der sogenannten Freiheit spürbar sind ${ }^{11}$. Vielleicht gelingt gerade deswegen Veteranyi der Spagat zwischen Ost und West, zwischen subjektivem Erleben und historischem Bewusstsein so gut, dass westliche Presse und Publikum sie als eines der «kraftvollsten und eigenwilligsten Talente» ${ }^{12}$ der zeitgenössischen Literatur feiern können. Aglaja Veteranyi vermag es, einerseits authentisch die Lebenswirklichkeit eines Flüchtlings aus dem östlichen Europa zu vermitteln und gleichzeitig aus diesem thematischen Fundus heraus eine verstörend-überzeugende, radikal experimentelle und trotz alledem durchaus unterhaltende Literatur hervorzubringen. Nach der Hysterie der Wanderjahre setzt mit der Kehrtwendung Aglaja Veteranyis in die Schweiz, an diesen topisch gewordenen Ort des europäischen Exils, ein unermüdlicher Prozess der Selbstfindung und Selbsterfindung ein, der sich schließlich in ein neues, schwer kanonisierbares Kapitel schweizerdeutscher Literaturgeschichte einschreibt ${ }^{13}$.

\title{
Muttersprache und Fremdsprache
}

\author{
Die anderen Kinder haben keine Angst, sie sprechen alle dieselbe Sprache. \\ Wir sprechen auch ihre Sprache, aber sie nicht unsere. \\ Ich kann in der fremden Sprache schon viele Wörter schreiben. Das Schreiben ist \\ aber anders als das Sprechen. Selbst Frau Hitz (Heimleiterin, LG) spricht anders, als \\ wir es in der Schule lernen. Ich frage mich, ob sie überhaupt so wie in der Schule \\ schreiben kann ${ }^{14}$.
}

Wesentlich für das Gelingen des literarischen Projekts ist die Entscheidung Veteranyis gewesen, die lingua franca der alltäglichen Lebenswirklichkeit künstlerisch in Anspruch zu nehmen und sie zur Darstellung peripherer Existenzformen, wie es diejenige rumänischer Flüchtlinge in der Schweiz ist, zu nutzen. Dabei kommt Veteranyi die Schweizer Vielsprachigkeit, insbesondere die Versetztheit des gesprochenen Schweizerdeutsch gegenüber der hochdeutschen Schriftsprache mit Sicherheit entgegen. Linguistische Aspekte wie diese rechtfertigen die Rezeption des Deutschen als neutralen, gleichzeitig artifiziellen und offiziellen schriftstellenden Grund, auf dem sich das Lebenstheater einer rumänischen Zirkusfamilie anschaulich entfalten lässt. Die deutsche Schriftsprache versinnbildlicht in diesem Fall den konventionellen Charakter einer jeden Sprache, wobei die Schweiz der Ort ist, an dem (auch linguistische) Fremdheit sesshaft werden kann. Darüber hinaus verrät eine Schriftsprache, anders als die gesprochene Sprache, nicht unmittelbar die Herkunft und nationale Zugehörigkeit eines Benutzers. Die Erfahrung sprachlicher Arbitrarität gestattet einerseits die Hinwendung zur Fremdsprache als künstlerisch zu handhabendes Werkzeug und andererseits die Loslösung von der Muttersprache. Mit einer Fremdsprache als poetisches Medium kann die Dichterin auf Wörter zurückgreifen, die für sie «frisch und 
ursprünglich $»^{15}$ sind. «Die Muttersprache ist wie das Blut in den Adern, sie fließt von allein. ${ }^{16}{ }_{1}:$ Die frühe Erfahrung von Mehrsprachigkeit und von territorialen Grenzüberschreitungen schließlich widerspricht dem Vorurteil, wonach die Bindung zu einer Sprache organischer Natur sei. Auf diese Weise wird Abstand von einer jeglichen adamistischen Sprachvorstellung, darüber hinaus von allen nationalen und ideologischen Klischees, die hiermit verbunden sind, genommen. Sprache wird entideologisiert ${ }^{17}$.

IN JEDER SPRACHE HEISST DASSELBE ANDERS. ${ }^{18}$

7 Eine solche Erkenntnis, wenn auch paradox, entlastet. Es geht nicht mehr darum, Wahrheit auszudrücken, vielmehr darum, Meinungen zu präsentieren sowie Begebenheiten und Gefühle sprachlich zu konkretisieren. Der Umgang mit und das Verständnis von Sprache werden pragmatisch.

Die Sesshaftigkeit wirkte sich auf meine Mutter und ihren Mann, den sie von unterwegs mitgebracht hatte, wie eine Schüttelkrankheit aus. Mich machte sie leise. Ich sprach leise, ging leise, dachte leise. Meine Gedanken waren in Schweigen verpackt ${ }^{19}$.

Die Autorin veranschaulicht in ihren Romanen anhand einer Ich-Erzählerin, die häufig als poetologische Identifikationsfläche dient, inwiefern der Erwerb des Deutschen als Umgangs- und Schriftsprache die konsequente Folge einer Entscheidung zur Sesshaftigkeit sowie mit dem Wunsch nach einer partiellen Integration in die soziale Umwelt und nach einem kulturellen Werteaustausch mit dem Gastland verbunden ist. Der Sprachwechsel führt die Autorin aus dem mündlich überlieferten Idiom einer Minderheit (ihrer Familie) in das Deutsch als Verkehrsprache der Mehrheit hinein. Damit wird ein kultureller Kontakt überhaupt erst möglich. Diese Entscheidung setzt allerdings nicht nur die Wahrnehmung des Anderen, sondern auch die Distanzierung vom Eigenen, die an sich selbst vollzogene Fremdwahrnehmung voraus: Der Rückzug auf sich selbst - in den Romanen verdeutlicht anhand einer Ich-Erzählerin, die selten direkt auftritt, sich vielmehr durch stumme Rede auszeichnet - gehen dem in der Literatur versuchten intersubjektiven und interkulturellen Dialog voraus. Dies bedeutet aber auch eine Entfernung von der eigenen Muttersprache. «Was nützt es mir, die fremde Sprache zu lernen, wenn meine Mutter sie nicht richtig versteht? ${ }^{20} »$ Auf der plot-Ebene der Romane wird die Abkehr von der Muttersprache mit der Abneigung der Tochter gegenüber der bildungsfeindlichen Mutter begründet: «Meine Mutter sagt, ich muss nicht in die Schule, das Wichtigste weiß ich schon. ${ }^{21} \gg$ Die Mutter vertritt die Ansicht, das Leben selbst sei der einzige notwendige Lehrmeister, und hat daher keine Skrupel, ihre Tochter bereits in jungen Jahren psychisch kaum zu bewältigenden Situationen auszusetzen. Die ambivalente Muttergestalt stellt auch beruflich eher ein Anti-Modell dar. Wie fast alle Mitglieder der Familie ist sie zwar nur eine Zirkusartistin, besteht aber darauf - eine Meisterin der Selbsttäuschung - als Künstlerin aufgefasst zu werden $^{22}$. Auf die Autorin Aglaja Veteranyi bezogen, betont die Wahl des Deutschen als Schriftsprache nicht zuletzt ihr Bestreben, aus dem fahrenden Artistenstand herauszutreten und eine anerkannte Schriftstellerin, eine wirkliche Künstlerin zu sein.

Auf der Ebene der Romanerzählungen ist das Verhältnis der Tochter zur eigenen Mutter ein stark belastetes, schwierig und von Komplexen beladen, nicht zuletzt deswegen, weil eine ausgleichende Vaterfigur fehlt ${ }^{23}$. Das von der Schwester erzählte Märchen von dem Kind, das in der Polenta kocht, stellt bildlich die Selbstopferung des Kindes zum Wohle der Mutter dar, ein Medea-Opfer, das auch als Sinnbild einer Sehnsucht nach Lieben und Geliebtwerden verstanden werden kann. Der italienische 
Begriff polenta steht hier - beinahe wie die feinere linguistische Variante aus dem Westen - als Übersetzung für das rumänische Wort mamaliga. Der Maisbrei ist das tägliche Brot des einfachen Volkes und eine der wichtigsten rumänischen Nationalspeisen überhaupt.

Meine Mutter und ich hatten keine Sprache miteinander. Nur Wörter ${ }^{24}$.

Alle Verwandten brachten mir Postkarten mit den Sehenswürdigkeiten der Stadt

und das gleiche Buch: Mihai Eminescu, Gedichte.

Ich konnte nicht Rumänisch.

Ich hatte einen Koffer voller Briefe, Fotos, Gratulationen und Arztrezepte.

Ein Koffer groß wie ein Land.

Die Sprache dieser Briefe wollte sich von mir nicht lernen lassen ${ }^{25}$.

Das Mutterland sowie die Muttersprache sind völlig von der Mutter, die stets davor steht, okkupiert und daher dem direkten Zugriff der Tochter entzogen. Die Selbstbehauptung der eigenen Identität muss daher mit einer Entfremdung von der Mutter und allem, wofür sie steht, beginnen. Während die Wahl der Schriftsprache Deutsch eine pragmatische ist, so verdeutlicht der gleichzeitige Verlust der Muttersprache das gestörte Verhältnis zur eigenen Herkunft sowie den Willen, aus dem familiären Kreis auszubrechen, die Nabelschnur zu durchtrennen. Ein Prozess der Selbstbildung und kulturellen Neuorientierung beginnt. Erst nachdem die Aneignung eines eigenen expressiven Werkzeugs erreicht wurde, ist ein identitätsbestimmender Rückblick auf die Herkunft sowie eine Selbstformulierung im neu gewonnenen Medium möglich. Die Erzählerin nimmt dabei die Rolle eines Dritten ein, wobei sie sich zugleich als Störfaktor und als Übersetzerin erweist ${ }^{26}$.

11 Für die Autorin Aglaja Veteranyi stellt der Erwerb und das Meistern der deutschen Sprache als Sprache der Mehrheit zunächst eine pragmatische Voraussetzung für das Gelingen des literarischen Projekts dar ${ }^{27}$. Die (deutsche) Sprache ermöglicht die kommunikative Verbindung zum sozialen Umfeld; ohne diese Mittlerin aber bliebe einerseits die Ausländerin ein Fremdkörper in ihrem Lebensumfeld, andererseits die Umgebung für sie unlesbar. Somit erhält das Schreiben auf Deutsch, aus der schweizerdeutschen Perspektive über ihre in der Fremde lebende Familie für die Autorin die Funktion einer versuchten Selbstverortung im kulturellen Grenzland. Bei näherem Hinsehen erweist sich die Autorin als eine Moralistin, eine Sittenbeobachterin, die sowohl die eigene Familie/Heimat als auch das Ausland/die Fremde aus einer halbverrückten Position heraus wahrnimmt und benennt ${ }^{28}$. Stellvertretend lässt sie ihre Erzählerin die Perspektive einer halben Außenseiterin einnehmen, die in der Literaturgeschichte nicht selten diejenige eines Narren oder auch eines Schelmen ist; diese in jeder Hinsicht halbverrückte Position wird durch den Sprachwechsel markiert. Die künstlerische Aneignung der herrschenden Sprache durch die Vertreterin einer sozialen Minderheit hat folglich beidseitig subversive Wirkungen, die über eine vorschnell $\mathrm{zu}$ vermutende bloße Anerkennung kultureller Machtverhältnisse hinausgehen ${ }^{29}$. Thematischer Fokus bleibt dabei fast ausschließlich der Nachvollzug der familiären Herkunft im Vergleich zur eigenen Persönlichkeitsentwicklung in der Fremde. Es ist der Versuch, sich schriftstellend im hic et nunc die Identität eines soweit wie möglich neutralen Filters, einer Membran für kulturelle Eigenwerte zu kreieren. Der literarischer Korpus Aglaja Veteranyis muss daher als ein selbständiger Versuch aufgefasst werden, im Austausch der Sprachen und kulturellen Traditionen, zwischen historischen und soziopolitischen Wirklichkeiten ein künstlerisches Territorium, das gleichzeitig ein existentielles Refugium bietet, zu 
definieren. Der Prozess des kreativen Schreibens ist für Veteranyi der wichtigste Weg, ihre nomadische Identität erzählerisch nachzuvollziehen, dabei als Künstlerin in einem artifiziell errichteten Provisorium, in einem Raum der Kunst wie in einer zweiten Haut zu überleben.

Im Roman ist es die ambivalente Mutterfigur selbst, die der Tochter die schriftstellende Tätigkeit ungewollt zusteckt:

Meine Mutter wird jemand finden, der ein Buch mit unserer Lebensgeschichte schreibt.

EISENTÜR UND TÜR ZUR FREIHEIT wird es heißen..$^{30}$

13 Subversiv ist das Werk von Aglaja Veteranyi in dreifacher Hinsicht: Zum einen schreibt sie mit ihren Erzählungen die - vielleicht unbedeutende - Geschichte einer rumänischen Flüchtlingsfamilie, die gleichzeitig auch ihre eigene Vorgeschichte ist, in die schweizerdeutsche Literatur ein. Derart konfrontiert sie das schweizerdeutsche Publikum mit den Eigenheiten einer kleinen Kultur. Zum anderen errichtet die Autorin jener Familie kein ehrenvolles Monument, sie dokumentiert vielmehr die moralische Ambivalenz, die im familiären Milieu vorherrscht. Schließlich markiert die von ihr beschriebene Zirkuswelt nicht nur in Bezug auf die neue Lebensumgebung Schweiz, sondern auch in Bezug auf das Herkunftsland Rumänien eine gesellschaftlich-kulturelle Randposition, womit jeglicher Ansatz eines nostalgischen Ursprungsmythos zerschlagen wird.

14 Aus den Texten erklingt vielmehr eine Erzählerstimme, die weltbedeutend ist, weil sie heimatlos wurde. Es ist ihre Aufgabe, Erinnerung an ein Dasein zu stiften, das sich ansonsten unbemerkt und zwischen allen Welten «auflösen» würde ${ }^{31}$. Das schriftlich gefasste Erzählen in derjenigen Schriftsprache, die am besten beherrscht und am wahrscheinlichsten rezipiert wird, erlaubt das Dokumentieren einer fragilen Existenz zwischen den Welten und die gleichzeitige Bezeugung einer individuellen Dichterstimme.

15 Diese Dichterstimme äußert sich in einer halb-fiktionalen, halb-dokumentarischen Schreibweise und versteht es darüber hinaus, sich vom Witz und von der Leidenschaft der spät erlernten Sprache zu speisen. Für die Autorin Aglaja Veteranyi, die im Zirkus aufgewachsen ist, lassen sich Wirklichkeit und Fiktion leicht verschränken.

UNSERE GESCHICHTE KLINGT BEI MEINER MUTTER JEDEN TAG ANDERS.

Wir sind orthodox, wir sind jüdisch, wir sind international!

Mein Großvater hatte eine Zirkusarena, er war Kaufmann, Kapitän, zog von Land zu

Land, verließ nie sein Dorf und war Lokomotivführer. Er war Grieche, Rumäne,

Bauer, Türke, Jude, Adliger, Zigeuner, Orthodoxer.

Meine Mutter trat schon als Kind im Zirkus auf, um ihre ganze Familie zu ernähren.

Ein andermal brennt sie gegen den Willen ihrer Eltern mit dem Zirkus und mit meinem Vater durch.

Das kostet meine Großmutter das Leben, obwohl sie in einer anderen Geschichte wegen unserer Flucht stirbt.

In allen Geschichten ist mein Großvater schon tot. ${ }^{32}$

16 Es handelt sich hierbei um eine Schreibweise, die sich weder einem starren Begriff des Faktischen noch einer reinen Fabulierlust anvertraut. Damit begibt sich die Erzählerin in Widerspruch zur mütterlichen Erzählweise: Die Erzählungen der Mutter, die durch Einfallsreichtum und Beweglichkeit beeindrucken, schweben bodenlos über jegliche Realität hinweg. Der Wille aber, sich schriftlich auszudrücken ist mit dem Wunsch nach einer gewissen Be- und Bodenständigkeit verbunden. 


\section{Tragisch-komische Miniaturen} synkretistischen Stil, wobei die deutsche Sprache die grammatikalische Struktur sowie das auf das Wesentliche und Notwendige beschränkte Vokabular, die rumänische Sprache hingegen bestimmte Redefiguren sowie eine Vorliebe für gewisse rhetorische Figuren liefert. Auch wenn die Erzählerin Rumänisch nicht zu schreiben vermag, so ist es doch ihr Anliegen, die Reden der Familienangehörigen ins Deutsche zu übertragen. Auf diese Weise wird die deutsche Sprache mit rumänischen Sprach- und Denkmustern durchsetzt: «Ganz Amerika! Ganz Europa! Engel gewöhnte sich die Familiensprache an. ${ }^{33}$ " Die Hyperbel ist das erkennbare rhetorische Prinzip dieser rumänisch geprägten Form des Sprechens und Erzählens; sie findet ihre Verkörperung in der Mutter selbst, an der alles übertrieben ist. Das Hyperbolische der Rede wiederum fällt umso mehr auf, als es im Medium einer lakonisch-zurückhaltenden Schriftsprache vermittelt wird. Aus diesem Widerspruch heraus entsteht nicht selten Ironie. Die durch Übertreibung verfremdete Rede kann aber auch neue poetische Bilder generieren. Dies ist zum Beispiel der Fall, wenn vom Herzen, das aus dem Mund wächst, gesprochen wird. Dazu müsste der Leser wissen, dass es im Rumänischen eine Redeweise gibt, die besagt, dass einem das Herz wächst, wenn man sehr gerührt ist. Das Wachsen des Herzens aus dem Mund herans stellt eine poetisch-expressive Übertreibung dar, die von der rumänischen Denk- und Redefigur angeregt wurde, im Weiteren aber mit artistischer Freiheit verändert wurde. Nichtsdestotrotz bleibt sie für einen deutschsprachigen Leser in ihrer Bedeutung erahnbar, auch wenn sie nicht als Redewendung erkannt, sondern als poetisches Bild aufgefasst wird. Man könnte sagen, die Erzählerin schenkt einer toten Metapher ein neues, ein transnationales Leben ${ }^{34}$.

Nach der Grenzöffnung war Onkel Petrus Besuch unvermeidlich. Die Schwestern fürchteten sich vor seinem Kommen. (...)

Am Flughafen lag Onkel Petru auf den Knien. Er hatte einen Rosenstrauß aus Bukarest mitgebracht und streckte ihn meiner Mutter, der Tante, dem Onkel und mir entgegen. Er hielt sich das seidene Tuch mit Kettenmuster, das ihm die Tante vor Jahren geschickt hatte, vor das Gesicht und weinte. Meine Mutter weinte, die Tante weinte. Der Onkel griff Onkel Petru unter den Arm und stellte ihn auf die Füße. Onkel Petru ließ sich wieder fallen. Ich lachte. Brauchen Sie Hilfe, fragte jemand.

Das kann nicht sein, rief Onkel Petru, das ist nicht wahr!

Steh auf, sagte meine Mutter, steh auf, du bist jetzt hier!

Petrule, steh auf, sagte die Tante, zu Hause kannst Du weitermachen.

Das kann nicht sein! Das glaub ich nicht! Das ist nicht wahr!

Alle paar Schritte ließ er sich auf den Boden fallen.

Beim Obst.

Beim Gemüse.

Bei den Delikatessen.

Jetzt bin ich hier, jetzt sag ich alles. Ich sag alles, was ich will!

Onkel Petru war ohne Zähne gekommen. ${ }^{35}$

Charakteristisch für die Erzählweise von Aglaja Veteranyi ist darüber hinaus die Verbindung von Tragik und Komik ${ }^{36}$. Ähnlich wie schon Eugène Ionesco, sieht auch Veteranyi keinen Widerspruch zwischen dem Tragischen und dem Komischen, erkennt vielmehr in der unauflöslichen Verbindung beider Elemente das eigentliche Pathos des 
Lebens ${ }^{37}$. Die von ihr porträtierten Gestalten sind lächerlich, kitschig und dennoch auf eigene Weise rührend.

Die Reisen zu den Verwandten sahen wie Auswanderungen aus. Die Wohnung wurde ein Lager für Rumänien, die Möbel wuchsen ineinander, in die aufeinandergelegten Teppiche sanken die Füße wie in Schlamm, durch Blumen, Papageien, Seehunde oder Bauchtänzerinnen hindurch.

Hier bricht man sich den Verstand, sagte ich. ${ }^{38}$

Typisch für die Rollenprosa von Aglaja Veteranyi ist schließlich das Wörtlich-Nehmen von deutschen oder von rumänischen Redewendungen. Die Inszenierung von Redemasken betont den performativen Charakter ihres Erzählens und weist auf seine Nähe zur Zirkus- und Theaterbühne hin ${ }^{39}$.

Die Tante und ich unterhielten uns in einer Sprache ohne Sprache.

Die Wärme meiner Tante drang in meine Haut ein. Wie eine Stimme. Eine Stimme aus Fleisch. ${ }^{40}$

Bei all dem artistischen Sprachspiel, den ironisch gebrochenen Redemasken und der hyperbolischen Inszenierungslust, scheint diese Literatur dennoch von einer großen Sehnsucht danach beseelt zu sein, eine jenseits allem Gerede sich ereignende kommunikative Situation herzustellen, die ein gegenseitiges Verständnis und menschliche Nähe zu fördern vermag. Das literarische Werk, in dem das Anziehende und das Furchterregende des Fremden thematisiert wird, formuliert zugleich vor allem in seinen stummen Passagen, in den Leerzeilen, die Suche nach einer körperlichexistentiellen Basis für die zwischenmenschliche Begegnung und den Dialog.

\section{Der gebürtige Ausländer}

Ein gebürtiger Ausländer hatte seine Schuhe verloren. Er hatte sie in seinem Haus liegengelassen und das Haus in einen Fluss weggeworfen. Oder hatte sich das Haus selbst hineingeworfen?

Der gebürtige Ausländer ging von Fluss zu Fluss.

Einmal fand er einen alten Mann unter Wasser mit einem Schild um den Hals: HIER HIMMEL.

Der Ausländer fragte: Wie, Himmel?

Der Alte zuckte die Achseln und zeigte auf das Schild.

Das Haus tauchte dann wieder auf, aber an einem ganz anderen Ort. Und wahrscheinlich war es ein anderes, denn es konnte sich an die Schuhe des Ausländers nicht erinnern.

Später verlor das Haus seine Tür ${ }^{41}$.

Die Erzählungen des Stiefvaters, der Mutter und der Schwester haben selbstreflexive Funktionen im Roman. Sie verdeutlichen zum einen individuelle Strategien zur Bewältigung und Interpretation des Schicksals. Die jeweilige kommunikative Ausdrucksform ist zugleich Inbegriff einer bestimmten Lebensform. Die Binnenerzählungen bieten somit der Erzählerstimme narrativische Modelle an und vermitteln gleichzeitig Lebensweisheiten. Die Stiefschwester erzählt grausame Kindermärchen. Die Mutter, die zwar die leibliche ist, aber wie die böse Stiefmutter aus der Märchenwelt agiert, verdreht in ihren Lügengeschichten die Wirklichkeit auf noch erfindungsreichere Art als Baron von Münchhausen. Der Stiefvater schließlich, der Clown ist, erzählt eine absurde Parabel. Diese Parabel, eine von Veteranyi zunächst selbständig erarbeitete Kurzgeschichte, die erst nachträglich in den Roman Polenta eingebaut wurde, verweist auf das Anliegen der Autorin, die historische 
Flüchtlingsexistenz künstlerisch und symbolisch zu erheben. Der Zigeuner und der Zirkusartist sind Figuren der ewig Fremden, der Wandernden ohne Aussicht auf Nomos. Als «gebürtiger Ausländer» entspricht der Wanderer einer modernen Auffassung der menschlichen Existenz. Über die Figur des «gebürtigen Ausländers» wird der Zustand der Migration, des Exils - «unsere Geschichte» - ontologisiert. Wenn aber die Migration universell ist, dann ist sie auch in jedem soziokulturellen Kontext vermittelbar.

Eine Analyse der sprachlichen Bildlichkeit bestätigt die Vermutung, dass die Texte von Veteranyi eine Existentialisierung der Migrationsliteratur anstreben. Häufig wiederkehrende Bilder wie die «der Kiste», «der Schachtel» und «des Koffers», die typisch für das metaphorische Repertoire von Migrantentexten sind, werden organischen Bildern des Obdachs und der Verhüllung, wie etwa "der Haut» gegenübergestellt. Die Universalisierung der Flucht- und Migrationserfahrung gehört zu der Strategie einiger Migrantenautoren, ihre literarischen Werke aus der Sparte der sogenannten Betroffenheitsliteratur, die primär politischen Zielsetzungen unterworfen ist, herauszuheben und mit avantgardistischen Formexperimenten kompatibel $\mathrm{zu}$ machen. Dies muss nicht zuletzt als eine Strategie verstanden werden, einen eigenen, transnationalen Literaturkanon aufzubauen: Dabei werden häufig solche Autoren zu einem intertextuellen Dialog versammelt, die innerhalb ihres jeweiligen Nationalkanons periphere Positionen einnehmen und damit Sprengkraft haben.

\section{Betroffenheitsliteratur und Avantgarde}

Bereits der Titel des Erstlingwerks von Aglaja Veteranyi ruft Fragen auf und verrät doch manches. Während das Bild des in der Polenta kochenden Kindes rätselhaft bleibt und zum Lesen des Romans auffordert, erinnert der sprechende Titel an den ebenfalls in medias res einsetzenden Titel des ersten Romans von Francesco Micieli Ich weiß nur, dass mein Vater große Hände hat (1988). Dabei hat dieser schweizerdeutsche Autor seine Wurzeln ebenfalls im Ausland ${ }^{42}$.

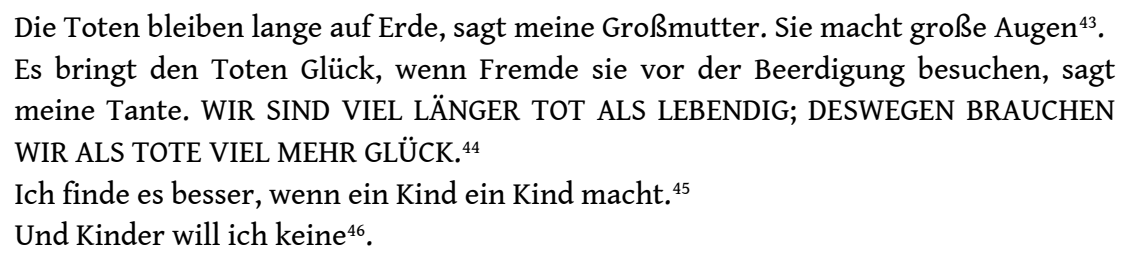

Die Nähe der Romane Aglaja Veteranyis zu denjenigen von Francesco Micieli als einem wichtigen Vertreter schweizerdeutscher Migrationsliteratur lässt sich thematisch, strukturell und im Detail nachweisen. Doch auch in Typographie und Aufbau gleichen sich die Werke und offenbaren auf den ersten Blick eine analoge, beinahe konkretvisuelle Ästhetik der <armen Materialien> und der $<$ Leerstellen ${ }^{47}$. Das Modell einer Migrationsliteratur, die Veteranyi und Micieli propagieren, inszeniert visuell und semantisch ihr Ringen um Sprache und Erzählung. In beiden Fällen werden Reduktionismus und Lakonismus $\mathrm{zu}$ den ästhetischen Prinzipien einer Literatur ernannt, in der sich «Sprachvermögen und Sprachangst [doppeln]» ${ }^{48}$.

Das Ringen um Sprache ist aber nicht nur für Migrantenautoren ein Thema. Veteranyi wie auch Micieli entdecken daher im Autor Peter Bichsel ein wichtiges literarisches Vorbild, auf den sowohl die sprechenden und oftmals tautologischen Titel als auch der Lakonismus als sprachkünstlerisches Verfahren in der schweizerdeutschen Literatur 
zurückgeht. Bereits in Bichsels 1964 erschienenen Erzählsammlung Eigentlich möchte Frau Blum den Milchmann kennenlernen wird «[...] Literatur gleichsam als Aussparung des zu Erzählenden» sichtbar ${ }^{49}$. Peter Bichsels Kindergeschichten führen darüber hinaus die bei Micieli und Veteranyi wieder auftretende Kinderperspektive ein, die mit scheinbarer Naivität die Logik der Sprache entdeckt und dekonstruiert, so etwa in der Erzählung «Ein Tisch ist ein Tisch» ${ }^{50}$.

Es ist diese Form von Literatur, die eine zwischen Autobiografie und Experiment changierende Migrationsliteratur vorzieht. Veteranyis Romanwerke ähneln kunstvollassoziativen Kombinationen von einzelnen poèmes en prose, in denen sogar die Leerstellen beredt sind. Andererseits wird im Kontext der Migrationsliteratur die Dialektik von Sagen und Nichtsagen(können), von Hüllen und Verhüllen über das rein Ästhetische und Erkenntnistheoretische hinaus gehend insofern verschärft, das Sprachspiel insofern existentiell aufgeladen, als das Thema der Identitätssuche und Identitätsformulierung zentral bleibt.

Die Suche nach der eigenen literarischen Identität im Schnittfeld west- und osteuropäischer Einflüsse zeigt sich bei einer weiteren Analyse der intertextuellen Bezüge im Werk von Veteranyi. Dabei erweist sich die innovatorische Kraft der häufig als peripher eingeschätzten kulturellen Phänomene, wie es die Literatur von Migranten ist. Autoren wie Aglaja Veteranyi, die gewissermaßen bei Null anfangen, greifen im Verlaufe ihres Selbstbildungsprozesses häufig auf Autoren zurück, die sich innerhalb ihrer jeweiligen literaturhistorischen Kontexte für eine Öffnung und Entsublimierung des literarischen Begriffs eingesetzt haben und deren Werke in Bezug auf nationale Kanons bis zum heutigen Zeitpunkt Grenzphänomene geblieben sind. Ein solcher Autor ist etwa der rumänische Prosaschriftsteller Urmuz. Urmuz betreibt zu Beginn des 20. Jahrhunderts eine radikale Auflösung literarischer Traditionen. Der Autor, der für die rumänische Avantgarde bis heute Kultstatus hat, gilt als Protodadaist. In seinen Kurzgeschichten spielt Urmuz mit dem Gegensatz von grammatikalischer Richtigkeit und semantischem Unsinn. Dabei nimmt er häufig Redewendungen wörtlich, spielt mit Zitaten aus Kunst und Alltag und setzt sich für die Vernichtung literarischer Klischees ein auf die Gefahr hin, wortlos zu bleiben.

Un jour, Groummer, sans prévenir Algazy, prit la brouette et partit tout seul à la recherche de loques et d'osselets, mais au retour, ayant trouvé par hasard aussi quelques restes de poèmes, feignit une maladie et, par-dessous l'édredon, les mangea tout seul furtivement ... En sentant cela, Algazy le suivit dans sa chambre, à l'intention sincère de lui tenir juste une toute petite harengue, mais observa, horrifié, dans l'estomac de Grummer, que tout ce qu'il y avait eu de bon dans la littérature avait été consommé et digéré. ${ }^{51}$.

Ein Meister der absurden Nullpunkt-Literatur und wohl ein weiteres Vorbild für Aglaja Veteranyi ist Daniil Charms. Charms pflegt den Infantilismus als ästhetische Strategie, um auf diese Weise und ähnlich wie Urmuz selbstverständlich gewordene Diskurse aus Kunst und Alltag in Frage stellen zu können. Bei ihm wird Literatur formal stark reduziert, manche seiner Kurzgeschichten sind nicht länger als fünf Verse.

Es war einmal ein rothaariger Mann, der hatte keine Augen und keine Ohren. Haare hatte er auch keine, so dass man ihn nur bedingt einen Rotschopf nennen konnte. Sprechen konnte er nicht, denn er hatte keinen Mund.

Eine Nase hatte er auch nicht.

Er hatte nicht einmal Arme und Beine. Und er hatte keinen Bauch, und er hatte keinen Rücken, und er hatte kein Rückgrat, und Eingeweide hatte er auch nicht. 
Überhaupt nichts hatte er! So dass man gar nicht versteht, von wem die Rede ist.

Besser, wir sprechen nicht mehr von ihm ${ }^{52}$.

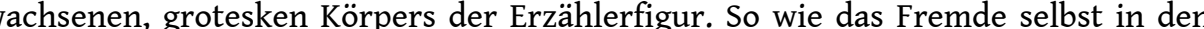
Texten Aglaja Veteranyis, so ist auch diese textuelle Feier von Hybridität ein ambivalentes Phänomen. Einerseits wird durchaus eine kosmopolitische Verbundenheit demonstriert. Andererseits spricht der Text ebenso eindeutig von drohenden Verlusten, Auflösungen, Zerbröckelungen. Es wird zerlegt, zerstückelt und verdaut. Zähne, Haut, Essen und Kochen betonen die kannibalistische SelbstKonsummation derjenigen Instanz, die die Erzählwelt generiert. Ein Opferungsritual scheint notwendig zu sein, um der Geschichte, dem literarischen Werk Bestand zu verleihen. Was geopfert wird, ist die Vorstellung einer apollinisch geschlossenen Erzähleridentität, die sich gänzlich vereinnahmen ließe.

Hier ist jedes Land im Ausland.

Der Zirkus ist immer im Ausland. Aber im Wohnwagen ist das Zuhause. Ich öffne die

Tür vom Wohnwagen so wenig wie möglich, damit das Zuhause nicht verdampft ${ }^{55}$.

Für das Kind, das im Wohnwagen aufwächst, sind sowohl «Heimat» als auch «Fremde» disloziierte Begriffe ${ }^{56}$. Das fremdsprachlich aufgewachsene Kind legt die im katachretischen Ausdruck «Wohnwagen» schlummernde Bedeutung wieder frei und erweckt erneut eine tote Metapher zum Leben: Der Wohnwagen, rulota auf Rumänisch, definiert den Ort einer nomadischen Existenz, für die nichts als die Veränderung selbst permanent sein kann. Das Kind identifiziert daher und konkretisiert die abstrakten Begriffe mit Gegenständen aus dem Alltag: der «Wohnwagen» ist folglich das «Zuhause» und die «Heimat». 

überleben und neue Wurzeln schlagen können. Hierbei muss es sich um transportable Güter, um immaterielles Kulturerbe handeln. Die Literatur Eminescus - des rumänischen Nationaldichters - erreicht dies nach Aglaja Veteranyi nicht. Die Riten der Nahrungszubereitung stellen in ihrer Sicht ein weitaus zukunftsträchtiges kulturelles Erbe dar.

Die gerösteten Auberginen meiner Mutter riechen überall wie zu Hause, egal, in welchem Land wir sind. (...)

WÄREN WIR ZU HAUSE, WÜRDE DANN ALLES WIE IM AUSLAND RIECHEN?

Mein Land kenne ich nur vom Riechen. Es riecht wie das Essen meiner Mutter ${ }^{57}$. Ich verstand die Muttersprache mit dem Geruch ${ }^{58}$.

In der Kochkunst drückt sich die Lebenslust und Vitalität der Mutter aus. Für die Mutter ist das Kochen keine alltägliche Sache, sondern ein sorgsam zelebriertes Ritual. Nach jedem wohl überstandenen Auftritt, wird das Weiterleben mit einer Hühnersuppe gefeiert. Dazu schlachtet die Mutter sogar das Huhn selbst. Dieses primitive Wissen ist es, das die Mutter in den Augen ihrer Tochter eigentlich auszeichnet ${ }^{59}$.

Roman zitierten Essenslisten weisen eine Dominanz rumänischer Gerichte vor Dabei ist die rumänische Küche selbst eine hybride Küche, die viele Einflüsse in sich aufgenommen und assimiliert hat: Gerade hierin besteht nach Meinung vieler Kenner ihr eigentümlicher und unverwechselbarer Geschmack ${ }^{60}$. In der Küche des Vielvölkerstaates Rumäniens spiegelt sich ihre wechselvolle Geschichte. So sind etwa die «gerösteten Auberginen» - vinete - mit den osmanischen Truppen von Indien aus nach Rumänien gelangt, haben auf dem Weg schrittweise Minze und andere Zutaten gegen Landesspezifischeres getauscht, aber nicht zuletzt ein Stück Geschichte hinzugewonnen.

Für die Erzählerin scheint die Küche die vielleicht einzig realisierbare Einverleibung der und in die Heimat. Das Kochen, ob als tägliche Versorgung der Lebenden oder als Wegbereitung für die Toten, wird als eine direkte Möglichkeit der Aneignung und Verdauung eines kulturellen Erbes vorgeschlagen. Während für die Eltern die Küche nur ein Index auf die Heimat darstellt, so identifiziert das Kind die Küche der Mutter mit der Heimat selbst, mit dem Mutterland, eine Verbindung, die mit jeder Hühnersuppe neu begründet wird.

Anlässlich des Todes der Tante wird ein Aspekt des orthodoxen Bestattungsrituals angesprochen - das Totenmahl bzw. der Totenkuchen.

Der Weizen muss in neun Wassern gewaschen werden, sagte Costel.

Warum?

Wegen den neun Himmeln.

Die Weizenkörner waren unruhig im Wasser, vielleicht wollten sie kein

Totenkuchen werden.

Im zehnten Wasser wird der Weizen gekocht, sagte er.

Neun Himmel?

Ja, für jeden Himmel einmal waschen.

Ich verstand die Muttersprache mit dem Geruch.

In der Küche der Tante standen Schränke auf den Schränken. Durch das Fenster schaute der Himmel in den Topf.

Er wartete.

Die Toten haben Hunger, sagte Costel. ${ }^{61}$

Das Kochen des Totenkuchens - coliva - ist ein Brauch, der auf heidnisch-antike Überbleibsel im orthodoxen Ritus verweist. Der Totenkuchen wird aus Weizen gekocht 
und steht für den Leib des Verstorbenen. Man hofft auf das Weiterleben des Toten, auf seine Wiedergeburt und gibt ihm daher den Weizen, ein Symbol für Fruchtbarkeit, mit auf den Weg.

Die Beherrschung kultureller Urakte wie Kochen und Bestatten verleihen auch der vulgären Existenz der rumänischen Flüchtlingsfamilie eine existentielle Tiefe: «Mit meiner Mutter gehe ich zum Fleischmarkt und mit meiner Tante zum Friedhof. ${ }^{62}$ » Mutter und Tante vertreten die beiden Seiten eines noch archaischen Kulturwissens, das in der rumänischen Kultur überlebt zu haben scheint. Sie betonen die enge Verzahnung von Leben und Tod, von Anfang und Ende.

40 Mit der Evokation der rituellen Nahrungszubereitung in Schwellenmomenten des Lebens, werden die Texte von Aglaja Veteranyi um eine Dimension des MythischArchaischen bereichert, die eine anthropologische Perspektive einführt, ohne sich allerdings in Volkstümlichkeit oder gar in Volkskunde zu verlieren. Für den westlichen Leser bedeuten diese Passagen vielmehr eine Initiation in poetische Mysterien. Mit ihrer Familienbeschreibung bringt die Erzählerin folglich nicht nur kulturelle Differenzen zur Sprache und ins Bewusstsein, sie überrascht auch mit einigen wenigen kulturellen Angeboten. Schließlich ist die Küche derjenige Bereich der Kultur, der sich Fremden am leichtesten mitteilen lässt. Dabei wird dieses mittelbare kulturelle Erbe von der Erzählerin mit dem gleichen Staunen entdeckt, das auch den westlichen Leser befällt.

41 Als eine Literatur der Schwellenerfahrung ist Migrationsliteratur in einem Zeitalter der Globalisierung von allgemeinem Interesse geworden. Daher sind auch transkulturelle Tendenzen in den Künsten in der heutigen globalisierten Welt auf eine paradigmatische Art und Weise bedeutsam. Migranten berichten darüber, wie man in der Fremde und unterwegs zurecht kommt, aber auch scheitern kann. Sie erschließen darüber hinaus die heutige Lebenswirklichkeit und den historischen Kontext. Die Vorstellung eines übernationalen europäischen Kulturraums gewinnt mit der Öffnung bzw. dem Wegfall der Grenzen zwischen Ost- und Westeuropa zunehmend an Realität. Die Migration von Ost nach West hat nicht zuletzt eine heterogene literarische Kultur gestiftet, die, wie das Beispiel Aglaja Veteranyis zeigt, anerkanntermaßen einen wesentlichen Beitrag zur zeitgenössischen deutschsprachigen Literatur leistet. Dabei fordert Migrationsliteratur den Begriff und die Institution einer «nationalen Literatur» auf radikale Art heraus.

Die Schweiz, die über eine Tradition der internationalen Neutralität verfügt und deswegen ein häufig genutztes Exilland ist, hat nichtsdestotrotz erst in den letzten Jahren Bemühungen dahingehend unternommen, die Literatur der sogenannten Secondos und Secondas - der Einwanderer in der zweiten Generation - offiziell als Teil der Schweizer Literatur anzuerkennen ${ }^{63}$. Ebenso ist in der Bundesrepublik, die sich nicht als Einwanderungsland begreift, erst im letzten Jahrzehnt eine Historisierung und Theoretisieren der Migrationsliteratur in Gang gekommen, etwa in den Werken von Sigrid Weigel, von Immacolata Amodeo oder von Carmine Chiellino ${ }^{64}$. In den Ursprungsländern der emigrierten Autorinnen ist eine solche Entwicklung aber oft noch gar nicht zu erkennen. Die rumänische Literaturgeschichtsschreibung z.B. pflegt nach dem Ende einer aufgesetzten, weil dogmatisch vorgeschriebenen Völkerfreundschaft das Verständnis einer organisch an der Nationalsprache gebundenen Literatur ${ }^{65}$. Eine Autorin wie Aglaja Veteranyi ist dann nicht mehr zu berücksichtigen, auch wenn ihr Werk, gerade weil es aus der Perspektive eines halben 
Außenseiters geschrieben ist, letztendlich wohl doch Aufschlussreicheres über Rumänien denn über die Schweiz mitzuteilen hat.

Der 〈Fall〉 Veteranyi stellt 〈die〉 schweizerdeutsche ebenso wie «die〉 rumänische Literatur in Frage und bringt beide gleichzeitig in eine Dialogsituation. Ein Ergebnis solch kulturell vermittelter Begegnungen könnte darin bestehen, im ästhetisch überzeugenden, literarischen Schaffen von Migranten eine transnationale Weltliteratur und eine gemeinsame, europäische Geschichte auszumachen.

Das Kind packt die Puppe in den Koffer.

Die Mutter packt das Kind in den Koffer.

Der Vater packt Mutter und Haus in den Koffer.

Das Ausland packt Vater mit Koffer in den Koffer.

Schickt alles zurueck.

Es verstecken sich im Wald:

1 Puppe

1 Kind

1 Mutter

1 Vater

1 Haus

2 Koffer

1 Flucht ${ }^{66}$

\section{NOTES}

1. Veteranyi, Aglaja: Das Regal der letzten Atemzüge, Stuttgart, Deutsche Verlagsanstalt, 2002, S. 62 und S. $65 f$.

2. Über die Bedeutung des von der Robert-Bosch-Stiftung jährlich verliehenen Preises für die Förderung der Migrationsliteratur im deutschsprachigen Raum informiert der Aufsatz von Esselborn, Karl: «Der Adelbert-von-Chamisso-Preis und die Förderung der Migrationsliteratur», in: Klaus Schenk, Almut Todorow, Milan Tvrdik (Hg.): Migrationsliteratur. Schreibweisen einer interkulturellen Moderne, Tübingen, Francke, 2004, S. 317-325.

3. Veteranyi, Aglaja: Geschenke. Ein Totentanz. Mit Holzschnitten von Jean-Jacques Volz, Zürich, Edition P. Petrej, 1999.

4. Veteranyi, Aglaja: Vom geräumten Meer, den gemieteten Socken und Frau Butter, München, Deutsche Verlagsanstalt, 2004.

5. Veteranyi, A.: Regal der letzten Atemzüge, A.a.O., S. 60.

6. Der erste Roman ist in vier Hauptteile gegliedert und präsentiert, trotz einiger analeptischer Erinnerungssprünge, chronologisch aufeinanderfolgende Lebensbeschreibungen aus der Sicht der Tochter als Kind. Die Darstellung umfasst die Zeitspanne von der Flucht der Familie aus Rumänien bis zu dem Augenblick ihrer Niederlassung in der Schweiz. Die brüchige Lebenserzählung wird im zweiten Roman weitergeführt, nun aus der Sicht der erwachsenen Tochter. Diesmal ist der dreiteilige Erinnerungsprozess erzähltechnisch anders organisiert: Eine 
Rahmenerzählung, die von dem qualvollen Tod der Tante handelt, schließt eine Retrospektive des Familienlebens in der Schweiz und nach 1989 nach dem Vorbild eines Familien-Foto-Albums in ihrer Mitte ein.

7. Veteranyi, A.: Regal der letzten Atemzüge, A.a.O., S. 76.

8. Veteranyi, A.: Warum das Kind in der Polenta kocht, A.a.O., S. 132.

9. Zunächst scheint nichts das Flüchtlings- und Artistenkind Aglaja Veteranyi für eine Karriere als schweizerdeutsche Autorin der Gegenwart zu prädestinieren, im Gegenteil: Die Herkunft, Rumänien, und der gewissermaßen exotische Werdegang, ihre Bildung als Flüchtling und Zirkusartist, decken sich kaum mit den Erwartungen, die man gängigerweise bezüglich einer Schriftstellerin hat. Darüber hinaus scheinen sich «Rumänien» und «Kultur» mittlerweile zu widersprechen. In der Zwischenkriegszeit hatte es zum ersten Mal eine Selbstbehauptung rumänischer Intelligenz und Kreativität im Westen gegeben, insbesondere in Frankreich, da sich die Rumänen als romanische Nation der französischen Kultur immer sehr nah fühlten und ihre Hauptstadt Bukarest als ein « Paris des Ostens » ansahen. Mit den Dada-Künstlern Tristan Tzara und Marcel Janco drangen (widerspenstige) Kulturträger rumänischer Herkunft auch in die Schweiz, genauer gesagt ins kosmopolitische Zürich hinein. Aglaja Veteranyi ist sich bewusst, dass sie einer anderen Generation rumänischer Migranten angehört. Nichtsdestotrotz wird sie an einem geradezu topisch gewordenen Ort des europäischen Exils sesshaft und die Kenntnis seiner vorausgegangenen literarischen Markierungen durch Migranten mag sie in ihrem eigenen Entschluss, eine künstlerische Existenz aufzubauen, bestärkt haben.

10. Veteranyi, A.: Warum das Kind in der Polenta kocht, A.a.O., S. 178.

11. Ebd., S. 28: «Zu Hause dürfen die Leute nicht einmal im Traum frei denken. [...] Die Fremden wollen uns aber auch schaden. [...] Meine Mutter traut niemandem. Ich muss das auch lernen.» Das totalitäre Regime im sozialistischen Rumänien stützte sich auf einem gefürchteten Überwachungssystem, der Securitate, das wesentlich dazu beitrug, jegliche Formen eines öffentlichen Lebens zu zerstören. Rumänien wurde asozialisiert. Der einzige Ort solidarischen Verhaltens war dann oft nur noch die Familie. Diese familiäre Schein-Artenbildung aber, die nicht nur in totalitären Regimen vorkommt, lässt die politischen Strukturen der Gesamtgesellschaft unangetastet und erzieht nicht selten zu Agoraphobie. Vgl. dazu Sennett, Richard: Verfall und Ende des öffentlichen Lebens. Die Tyrannei der Intimität, Frankfurt/Main, Fischer, 10. Aufl., 1999. S. $389 f f$.

12. Kunisch, Hans-Peter: «Aglaja Veteranyi: Das Regal der letzten Atemzüge. Rezension» In: Süddeutsche Zeitung vom 22.10.2002.

13. Obwohl seit Jahrzehnten im Westen verweilend und seit vielen Jahren schon literarisch tätig, werden manche <Fälle> wie Aglaja Veteranyi erst nach der Wende publikumswirksam rezipiert. Als Gründe hierfür wären verstärkte Migrationswellen von Ost nach West, eine zunehmende Neugierde westlicherseits bezüglich der östlichen Nachbarn, das gemeinsame Interesse an kulturellen Schnittmengen, an Modellen für eine europäische Identität zu nennen. Für die Literaturwissenschaft gilt, dass erst in den späten 1980er Jahren mit einer ernsthaften Öffnung der Germanistik hinsichtlich der Historisierung und Theoretisierung sogenannter Migrationsliteratur begonnen wurde.

14. Veteranyi, A.: Warum das Kind in der Polenta kocht, A.a.O., S. 100.

15. Foster, Leonard: Dichten in fremden Sprachen. Vielsprachigkeit in der Literatur, München, Francke, 1974, S. 100.

16. Veteranyi, A.: Warum das Kind in der Polenta, A.a.O., S. 151.

17. An dieser Stelle muss angemerkt werden, dass es in Bezug auf die rumänische Rezeption westlicher Kultur zwei miteinander konkurrierende Modelle mit Vorbildcharakter gab und weiterhin gibt: das französische und das deutsche Modell. Anders aber als ein Ion Luca Caragiale im 19. Jahrhundert, scheint Aglaja Veteranyi keine Theorie der deutschen Sprache und der deutschen Kultur zu haben, insofern ist der Gebrauch des Deutschen für sie pragmatisch 
motiviert. Eine Verbindung zu Caragiale ließe sich dennoch über die gemeinsame Vorliebe für den Sprachwitz und für satirische Inszenierungen herstellen. Caragiale, ein Klassiker der rumänischen Literaturgeschichte, war einer der leidenschaftlichsten Anhänger des deutschen Modells; er hatte sich gleichzeitig vorgenommen, das Balkanisch-Widersprüchliche in der rumänischen Gesellschaft scharfzüngig zum Vorschein zu bringen. Caragiale ist schließlich ein wichtiges Vorbild für Eugène Ionesco gewesen, der das satirische Sittenbild existentialisierte.

18. Veteranyi, A.: Warum das Kind in der Polenta, A.a.O., S. 87.

19. Veteranyi, A.: Das Regal der letzten Atemzüge, A.a. O., S. 75.

20. Veteranyi, A.: Warum das Kind in der Polenta, A.a.O., S. 100.

21. Ebd., S. 62.

22. Mit ihrer lebensgefährlichen Nummer (sie hängt an den eigenen Haaren in der Zirkuskuppel) ist sie die eigentliche Attraktion im Zirkus. Von ihren seidenen Haarspitzen hängt die Existenz der ganzen Truppe ab.

23. Angst ist das dominierende Kennzeichen dieser Mutter-Tochter-Beziehung. Die Tochter hat zunächst Angst um die Mutter. Sie hat aber auch Angst vor der Mutter, vor deren psychischemotionalen Labilität, ihrer Unzuverlässigkeit, ihrer moralischen Inkonsequenz. Schließlich ist es die Angst vor der Dominanz der Mutter, davor, das mütterliche Vorbild niemals erreichen zu können. Liebe schlägt in Feindschaft um: Das Kind ertappt sich dabei, dass es sich eine Entlastung seines eigenen Bewusstseins und damit das Verschwinden der Mutter herbeiwünscht. Dieser Wunsch wiederum produziert Schuldgefühle, die sich erneut gegen das Kind selbst richten.

Der fehlende, unbekannte Vater ist ein Indiz auf die Tatsache, dass das Vaterland (patrie auf Rumänisch) für die Tochter uneinholbar bleibt.

24. Veteranyi, A.: Regal der letzten Atemzüge, A.a.O., S. 71.

25. Veteranyi, A.: Regal der letzten Atemzüge, A.a.O., S. $91 \mathrm{f}$.

26. Zur Figur des Dritten vgl. Arne Höcker, Jeannie Moser, Philippe Weber (Hg.): Wissen. Erzählen. Narrative der Humanwissenschaften, Bielefeld, transcript, 2006.

27. Obschon ihre literarischen Werke um einen ganz eigenen thematisch-motivischen Kern kreisten, blieb Aglaja Veteranyi keine singuläre oder vereinzelte Erscheinung, im Gegenteil, sie war maßgeblich an dem Erblühen neo- avantgardistischer Literaturkonzepte in Zürich beteiligt. Aglaja Veteranyi schloss sich der 1992 von Perikles Monioudis, Peter Weber und Ruth Schweikert gegründeten und in Kritikerkreisen Ansehen genießenden Jungautorengruppe «Netz»an. 1993 gründete sie zusammen mit René Oberholzer die Experimentaltruppe «Die Wortpumpe», die Sprachspiele der beiden Autoren auf die Bühne brachte. All dies spricht dafür, dass Veteranyis ästhetisches Interesse auf eine an dadaistische und futuristische Vorbilder erinnernde Synthese von Sprachkunst und Theaterspiel zielte. Dies wird auch durch das Projekt «Die Engelsmaschine» bekräftigt, das Aglaja Veteranyi 1996 zusammen mit Jens Nielsen initiierte und bei dem es sich um eine Performance-Theatergruppe handelte, die vornehmlich Texte der Autorin inszenierte.

28. Es fällt auf, dass die thematische Auseinandersetzung mit der Schweiz einen geringeren Stellenwert einnimmt, als die Beobachtung der eigenen Familie. Die Schweiz wird als urbaner Rahmen evoziert und taucht ansonsten in Details auf, die metonymisch auf die gesellschaftlich benachteiligte Randposition der Flüchtlingsfamilie hinweisen, so wie etwa im Bild des «Wohnblocks», das anstelle des ersehnten großen Hauses das neue Heim der Familie darstellt. Dieser thematische Fokus auf die eigene Familie hat sicherlich auch damit zu tun, dass sich der Autorin dort starke, schicksalhafte Geschichten zum Erzählen anbieten.

29. Vgl. hierzu Amodeo, Immacolata: ¿Die Heimat heißt Babylon〉. Zur Literatur ausländischer Autoren in der Bundesrepublik Deutschland, Opladen, Westdeutscher Verlag, 1996.

30. Veteranyi, A.: Warum das Kind in der Polenta kocht, A.a.O., S. 53.

31. Ebd., S. 33: «Das Abbauen des Zirkuszeltes ist überall gleich, wie ein großes Begräbnis, immer in der Nacht, nach der letzten Vorstellung in einer Stadt. [...] Bei mir löst sich alles auf, und es geht ein Wind durch mich hindurch.» 
32. Ebd., S. 57.

33. Veteranyi, A. : Das Regal der letzten Atemzüge, A.a.O., S. 70.

34. Zum Begriff des Transnationalen vgl. Welsch, Wolfgang: «Transkulturalität. Zur veränderten Verfasstheit heutiger Kulturen», in: Zeitschrift für Kulturaustausch 45, Nr. 1/1995, S. 39-44.

35. Veteranyi, A.: Das Regal der letzten Atemzüge, A.a.O., S. 94 und S. 96.

36. Eine weitere Künstlerfigur in der Familie ist Onkel Petru, der homosexuelle Maler aus Bukarest. Onkel Petru wurde nach der Flucht seiner beiden Schwestern - der Mutter und der Tante - stellvertretend von der Securitate in die Mangel genommen. Er kam für viele Jahre ins Gefängnis, wo er gefoltert und ihm dabei die Zähne ausgeschlagen wurden.

Die Zähne sind ein in den Texten wiederkehrendes Motiv; als «schlechte Zähne» verweisen sie metonymisch auf die Armut und Verwahrlosung Rumäniens.

37. Ionesco, Eugène : Notes et contre-notes, Paris, Gallimard, 1966, S. 61 : «Je n'ai jamais compris, pour ma part, la différence que l'on fait entre comique et tragique. Le comique étant l'intuition de l'absurde, il me semble plus désespérant que le tragique. Le comique n'offre pas d'issue.»

38. Veteranyi, A. : Das Regal der letzten Atemzüge, A.a.O., S. 90.

39. Veteranyis künstlerische Laufbahn beginnt zunächst mit einer Theaterausbildung an der «Schauspiel Schule Zürich». Die Schauspiel Schule wurde 1950 von Hannes Becher gegründet, der ein wichtiger Mentor für Aglaja Veteranyi war. Später übernahm sie die Co-Leitung der Schule. Viele ihrer Texte, so auch der Roman Warum das Kind in der Polenta kocht, wurden für die Bühne adaptiert.

Auffällig ist bei Veteranyi das Fehlen von konkreten zeitlich-räumlichen Koordinaten. Die Geschehnisse finden gleichermaßen auf einer leeren und dunklen Bühne statt, auf der von Zeit zu Zeit ein Lichtschein bestimmte Ecken mit all den darin befindlichen Figuren und Requisiten temporär beleuchtet. Die erzählerische Wirklichkeit muss als eine innere Landschaft gedacht werden; die erinnerten Geschehnisse ereignen sich retrospektiv und in einer neuen Sprache übersetzt im Bewusstsein der Erzählerin.

40. Veteranyi, A.: Regal der letzten Atemzüge, A.a.O., S. $125 \mathrm{f}$.

41. Veteranyi, Aglaja: Vom geräumten Meer, den gemieteten Socken und Frau Butter, A.a.O., S. 94.

42. Francesco Micieli ist Italo-Albaner. Er wurde 1956 in eine albanesische Familie in Kalabrien hineingeboren. Seine Eltern flohen vor der Armut in der Heimat und gelangten als Arbeitskräfte in die Schweiz. Micieli studierte, wie später Aglaja Veteranyi, ebenfalls Schauspielerei; er wurde Theaterautor und Regisseur. Der oben zitierte Roman ist Teil einer Trilogie, zu der im weiteren die Romane «Das Lachen der Schafe» (1989) und «Meine italienische Reise» (1996), und die aus unterschiedlicher Erzählperspektive Auswanderung, Alltag im Ausland, sowie Rückkehr in die Heimat einer Migrantenfamilie aus Kalabrien beschreibt. Der oben zitierte erste Roman ist wie Veteranyis Romanwerke aus der Ich-Perspektive eines Kindes geschrieben.

43. Micieli, Francesco: Ich weiß nur, dass mein Vater große Hände hat, Bern, Kurt Salchli, S. 11.

44. Veteranyi, Aglaja: Warum das Kind in der Polenta kocht, A.a.O., S. $60 f$.

45. Micieli, F.: Ich weiß nur, dass mein Vater große Hände hat, A.a.O. S. 46.

46. Veteranyi, A.: Warum das Kind in der Polenta kocht, A.a.O., S. 117.

47. So etwa aufgrund der Leerzeilen zwischen den Sätzen, der nur halb- oder dreiviertel bedruckten Seiten, oder auch der fehlenden Kapitelüberschriften.

48. Reinacher, Pia: «Auch Augenklimpern kostet. Aglaja Veteranyis Protokoll der Warenwelt», in: Frankfurter Allgemeine Zeitung vom 03.12.2002.

49. Schnell, Ralph: Geschichte der deutschsprachigen Literatur seit 1945, Stuttgart, Metzler, 1993, S. 517.

50. Vgl. Bichsel, Peter: «Ein Tisch ist ein Tisch.», in: Kindergeschichten, Darmstadt, Luchterhand, S. 18-27. Von der Tautologie ausgehend, führt der Mann in einem spielerischen Selbstexperiment die Arbitrarität der Zeichen vor und kreiert gleichzeitig seine eigene Sprache, die ihn allerdings so weit wie möglich vom kommunikativen Konsensus entfernt und damit in den Wahnsinn treibt. 
51. Urmuz: « Alcazy et Grummer », in : Pages Bizarres, Timisoara, Hestia, 1998, S. 80.

52. Charms, Daniil: « Blaues Heft Nr. 10 », in: Fälle, Stuttgart, Reclam, S. 5.

53. «Oberiu» ist eine freie Zusammensetzung der Anfangsbuchstaben von «Objedinenije realnowo iskusstwa». Die Rumänisierung der Gruppenbezeichnung fällt auf. Dies wird üblicherweise mit der Tendenz zur Selbst-Exotisierung interpretiert, es bleibt aber zu vermuten, dass die Mitglieder der Gruppe Kenntnis von der zu jenem Zeitpunkt bereits publizierten absurden Dichtung Rumäniens hatten.

54. Ebd., S. 67.

55. Veteranyi, A.: Warum das Kind in der Polenta, A.a.O., S. 10.

56. Vgl. Schifferle, Judith: «Zwei Autorinnen schauen fern. Aglaja Veteranyi und Herta Müller zwei aus Rumänien stammende deutschsprachige Autorinnen im Vergleich», in: Trans. InternetZeitschrift für Kulturwissenschaften, Nr. 15, April 2004. (Stand: 25.01.2005)

57. Veteranyi, A.: Warum das Kind in der Polenta kocht, A.a.O., S. 10.

58. Veteranyi, A.: Regal der letzten Atemzüge, A.a.O., S. 9.

59. Veteranyi, A.: Warum das Kind in der Polenta kocht, A.a.O., S. 17: «In der Morgendämmerung steht meine Mutter auf und beginnt zu kochen, rupft das Huhn und hält es über die offene Gasflamme. Meine Mutter kauft am liebsten lebendige Hühner, weil sie am frischesten sind. Im Hotel schlachtet sie das Huhn in der Badewanne.» und S. 70: «DAS SCHÖNSTE: Wenn wir nach der Vorstellung zusammen essen.»

60. Vgl. hierzu das geistreiche Kochbuch des kürzlich verstorbenen Roman, Radu Anton: Savoureuse Roumanie, Paris, Éditions Noir sur Blanc, 2004.

61. Veteranyi, A.: Regal der letzten Atemzüge, A.a.O., S. 9.

62. Veteranyi, A.: Warum das Kind in der Polenta, A.a.O., S. 60. Es lässt sich behaupten, dass der erste Roman Veteranyis ein Buch über die Mutter, der zweite Roman ein Buch über die Tante ist. 63. Vgl. hierzu Rothenbühler, Daniel: «Im Fremdsein vertraut. Zur Literatur der zweiten Generation von Einwanderern in der deutschsprachigen Schweiz: Francesco Micieli, Franco Supino, Aglaja Veteranyi.», in: Migrationsliteratur. A.a.O., S. 51-78.

64. Vgl. hierzu Weigel, Sigrid: «Literatur der Fremde - Literatur in der Fremde.», in: Briegleb, Klaus / Weigel, Sigrid (Hg.): Gegenwartsliteratur seit 1965 (Hansers Sozialgeschichte der deutschen Literatur Bd. 12), München/ Wien, Hanser, 1992, S. 182-193. Chiellino, Carmine: Am Ufer der Fremde. Literatur und Arbeitsmigration 1870-1991, Stuttgart/Weimar, Metzler, 1995.

65. So lässt sich in der 2005 erschienenen umfassenden Geschichte der zeitgenössischen rumänischen Literatur weder ein Eintrag zur Minderheitenliteratur in Rumänien noch zur fremdsprachlichen Literatur von Exilrumänen (abgesehen von derjenigen Ciorans, Eliades und Ionescos) finden. Vgl. Stefanescu, Alex: Istoria literaturii române contemporane. 1941-2000. Bucuresti, Editura de scris, 2005. 66. Veteranyi, A.: Vom geräumten Meer, den gemieteten Socken und Frau Butter. A.a.O., S. 49.

\section{RÉSUMÉS}

Aglaja Veteranyi (1962-2002), die schweizerdeutsche Autorin rumänischer Herkunft, behandelt in ihren Prosawerken die autobiographisch inspirierten und existentiell zugespitzten Themen der Migrantenliteratur in der Form des literarischen Minimalismus. Veteranyi bedient sich dabei fast ausschließlich der deutschen Schriftsprache, wobei der deutsche Ausdruck sich bei ihr durch lakonische Kürze, Einfachheit und Schmucklosigkeit auszeichnet. Insofern Deutsch aber als 
Medium der poetischen Inszenierung und Übersetzung familiärer Reden dient, kommt es zu einer Durchsetzung des Textes mit Metaphern, die auf rumänische Sprach- und Denkfiguren zurück zu gehen scheinen. Diese rhetorischen Elemente sind grundsätzlich vom Prinzip des Hyperbolischen bestimmt und daher häufig grotesk. Veteranyi betont, dass es weder eine Heimkehr nach der Flucht noch eine authentische Sprache geben kann, deren Erhalt eine immerwährende Bindung an das Mutterland gewährleisten könnte. Mit ihren eigenen Texten versucht sie eine Gratwanderung im Schnittfeld von ost- und westeuropäischer Literatur- und Kulturgeschichte. Im semi-fiktionalen Raum des literarischen Textes wird die Konstituierung einer hybriden Identität beschworen, die nationale Kanonbegriffe sprengt.

Aglaja Veteranyi (1962-2002) est une écrivaine suisse-allemande d'origine roumaine. Dans ses œuvres en prose écrites dans un style minimaliste, elle traite les sujets autobiographiques et existentiels qui caractérisent la littérature des migrants. Vétéranyi se sert uniquement de l'allemand comme langue écrite. L'allemand de Veteranyi est une langue simple et laconique. En citant les discours familiers, l'auteur traduit cependant quelques expressions roumaines dans ses textes. Ces images poétiques d'origine roumaine sont souvent hyperboliques et contribuent aux aspects grotesques des romans. Aglaja Veteranyi ne croit pas qu'un retour aux origines soit possible : À son avis, la fuite est toujours définitive et éternelle. De plus, il ne peut pas y avoir de langue authentique qui serait capable de maintenir une relation étroite avec le pays maternel qui a été perdu. Dans ses propres œuvres, l'auteur se situe plutôt dans une zone frontière entre la tradition littéraire (et culturelle) de l'Europe de l'ouest et celle de l'Europe de l'est. C'est dans l'espace sémi-fictionel du texte littéraire que l'auteur entreprend d'établir une identité hybride qui défie tous les canons nationaux.

\section{INDEX}

Mots-clés : émigration roumaine, littérature de la migration oeuvrecitee Das Regal der letzen Atemzüge, Geschenke. Ein Totentanz, Vom geräumten Meer den gemieteten Socken und Frau Butter, Warum das Kind in der Polenta kocht

\section{AUTEURS}

\section{LAURA GIESER}

Martin-Luther-Universität Halle-Wittenberg 